\title{
Fuzzy adaptive teaching learning-based optimization strategy for the problem of generating mixed strength $t$-way test suites
}

\begin{abstract}
The teaching learning-based optimization (TLBO) algorithm has shown competitive performance in solving numerous real-world optimization problems. Nevertheless, this algorithm requires better control for exploitation and exploration to prevent premature convergence (i.e., trapped in local optima), as well as enhance solution diversity. Thus, this paper proposes a new TLBO variant based on Mamdani fuzzy inference system, called ATLBO, to permit adaptive selection of its global and local search operations. In order to assess its performances, we adopt ATLBO for the mixed strength $t$-way test generation problem. Experimental results reveal that ATLBO exhibits competitive performances against the original TLBO and other meta-heuristic counterparts.
\end{abstract}

Keyword: Software testing; $t$-way testing; Teaching learning-based optimization algorithm; Mamdani fuzzy inference system 